\title{
Germanium Nanowires as Spectrally-selective Photodetectors in the Visible-to-Infrared
}

\author{
Amit Solanki ${ }^{1}$, Hyunsung Park ${ }^{1}$ and Kenneth B. Crozier ${ }^{1,2,3, *}$ \\ ${ }^{1}$ School of Engineering and Applied Sciences, Harvard University, Cambridge, MA 02138, USA \\ ${ }^{2}$ Department of Electrical and Electronic Engineering, University of Melbourne, VIC 3010, Australia \\ ${ }^{3}$ School of Physics, University of Melbourne, VIC 3010, Australia \\ *kcrozier@unimelb.edu.au
}

\begin{abstract}
We experimentally demonstrate arrays of vertical Ge nanowires as spectrally-selective photodetectors at visible to infrared wavelengths. Measurements reveal that the external quantum efficiency spectra of fabricated devices vary with the radius of the constituent nanowires.
\end{abstract}

OCIS codes: (040.5160) Photodetectors; (110.4234) Multispectral and hyperspectral imaging; (220.4241) Nanostructure fabrication.

Nanowires (NWs) present interesting possibilities as photodetectors because the power they absorb can be tailored by appropriate choice of their geometry. NW-based photodetectors have been primarily studied in the visible wavelength range [1-3], with the short -wave-infrared (SWIR) range being mostly unexplored, despite important defense, medical and consumer applications. This is mostly due to the lack of materials that can efficiently absorb SWIR light. Current state of the art SWIR photosensors are based on III-V materials which can be toxic and expensive. Furthermore, for multispectral imaging at SWIR wavelengths, the current approach [4] involves mounting broadband image sensors on bulky spectrometers. In our recent work [5], we suggested the use of germanium NWs as photodetectors due to their potential for low cost, SWIR sensitivity and ease of nanostructuring as a CMOS compatible material. A novel approach was proposed by using Ge NWs nanofabricated on Si substrate as spectrally sensitive absorbers to allow for light absorption from visible to SWIR. In such a device the incident light is split by SWIR absorption in Ge NW array photodetector and VIS absorption in Si substrate photodetector. We then showed that the reflectance spectrum of Ge NW arrays could be tuned by changing the diameter of all NWs in the array. The reflectance and absorption being complementary, the study highlighted the feasibility of Ge NW based photodetectors with spectrally selective absorption. In this submission, we show the proof of concept of a Ge NW array based active photodetectors with absorption spectra tuned in SWIR region by varying the array diameter. The work takes us a step closer to realization of image sensor that would achieve visible-to-SWIR multispectral imaging.

a)

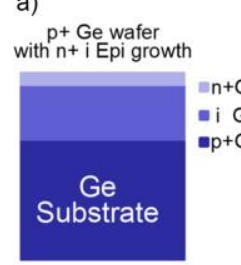

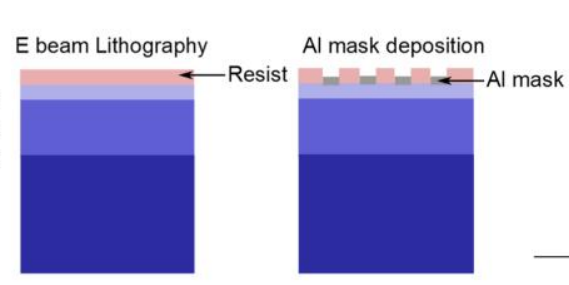

b)

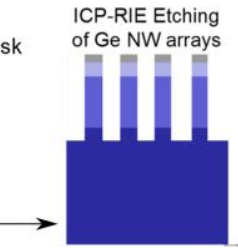

c)

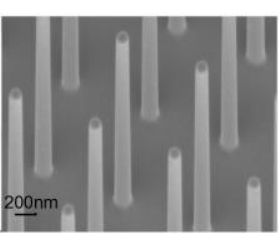

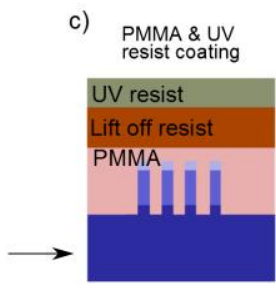

d)

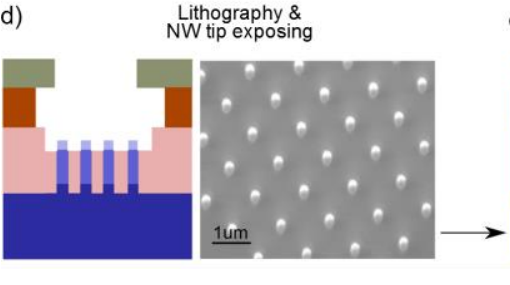

e) ITO Sputtering

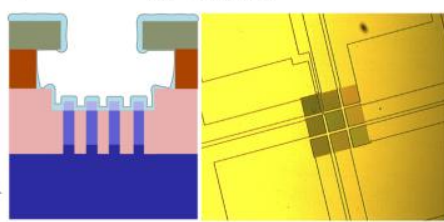

f)
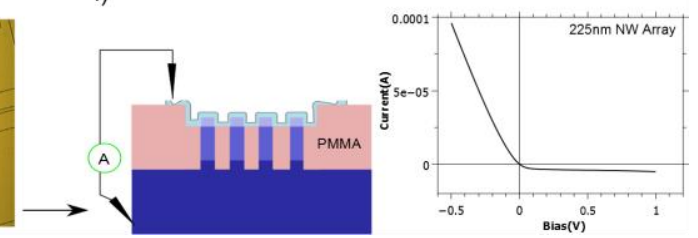

Figure 1. Schematic of the nanofabrication process. a) Lithography with Al mask deposition is performed on Ge wafer with p-i-n stack. b) Nanowires are etched using $\mathrm{Al}$ as mask. c) PMMA holding layer and UV photoresist are spun coated d) After lithography and development the nanowire tips are exposed by $\mathrm{O}_{2}$ plasma etching. e) Next ITO is sputtered. f) Lift off is done and I-V is measured between ITO and substrate.

The starting substrate was a Ge p+ wafer with epi-grown intrinsic Ge $(1.5 \mu \mathrm{m})$ and $\mathrm{n}+\mathrm{Ge}(300 \mathrm{~nm})$ layers. NWs were etched to heights of $2 \mu \mathrm{m}$ using the method we previously described (Fig. 1a-b, [5]). It is important to note that 
the intrinsic section is fully embedded in the NWs. This mitigates the possibility of the collecting carriers photogenerated in the bulk Ge substrate. The array was then embedded in polymethylmethacrylate (PMMA) by multiple spin coating steps (Fig. 1c). A bilayer resist next applied and patterned by optical lithography to produce fan-out contacts to the individual NW arrays. After development, $\mathrm{O}_{2}$ plasma etching was performed to etch the PMMA and expose the NW tips. Once the exposed tip heights of $250 \pm 50 \mathrm{~nm}$ were obtained, ITO layer was sputtered and lift off was performed to obtain contacted arrays. PMMA away from the contacts was carefully removed from the substrate with a razor blade to expose the $\mathrm{p}+\mathrm{Ge}$ substrate which served as the bottom electrode. Electrical connections were made, and the measured current-voltage plot (Fig. 1f) confirmed diode behavior.
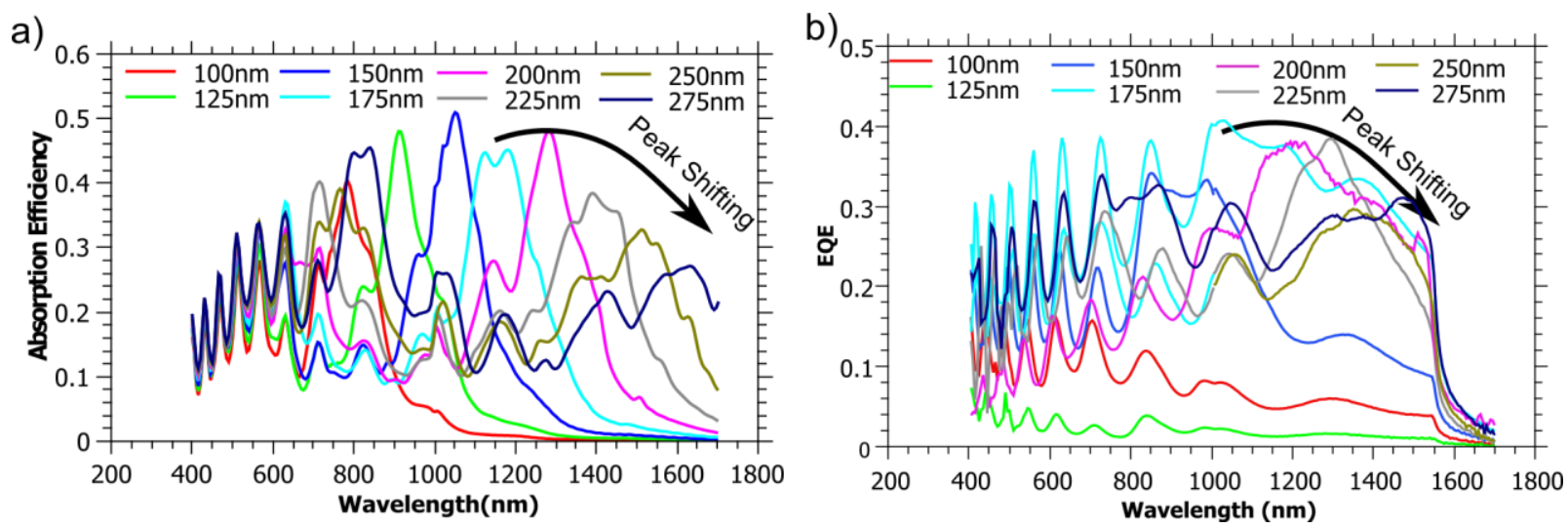

Figure 2. Simulated absorption spectra and measured EQE of arrays of Ge NWs with different diameters.

Electromagnetic simulations were performed using the finite difference time domain method (FDTD, Lumerical). The NWs were chosen to have heights of $2 \mu \mathrm{m}$, and diameters of 100 to $275 \mathrm{~nm}$. Periodic boundary conditions with a $1 \mu \mathrm{m} \times 1 \mu \mathrm{m}$ unit cell were used. To estimate the light absorption in the active region of the fabricated device, we found the absorption from the intrinsic region from our simulation results. Simulations results shown in Fig. 2a predict peaks in absorption spectra, whose position shifts to longer wavelength with increasing NW diameter. The peaks in absorption correspond to reflection dips in our previous study [5], and are due to wavelength dependent resonant light absorption in guided modes of the NW. The spectral responses of the fabricated devices were found from measurements with a homemade set-up. A tungsten halogen lamp was used as a light source along with monochromator (MC) to obtain light with wavelengths from $400 \mathrm{~nm}$ to $1700 \mathrm{~nm}$. A $1000 \mathrm{~nm}$ high pass filter was mounted before the exit slit of the MC for wavelengths above $1000 \mathrm{~nm}$ to eliminate the second diffraction order. A pinhole of $200 \mu \mathrm{m}$ diameter was mounted after the exit slits of the MC. The light from the pinhole passed through a beamsplitter and was focused by an objective lens (10X magnification) onto the NW array. The array was imaged using a CMOS camera mounted on the same beamsplitter. Two translation microprobes were then used to make contact with the top ITO and back substrate contacts. A chopper mounted in the path of the incident beam right after the pinhole triggered a lock-in amplifier which measured the photocurrent from the sample. To obtain the device responsivity, the sample was replaced with calibrated Si (400 to $1000 \mathrm{~nm}$ range) and InGaAs (1000 to $1700 \mathrm{~nm}$ range) photodiodes. The NW responsivity was then obtained by dividing the NW photocurrent by the photodiode photocurrent and finally multiplying by calibrated responsivity of the photodiode. The external quantum efficiency (EQE, Fig. 2b) was calculated from the measured responsivity (see e.g. [3]). We see that the experiment, as predicted by simulation, show a redshift in peaks with increasing NW diameter. It should be noted that we simulate untapered NWs with the diameter taken from the design values used for E-beam pattering of the array. The actual nanowires are tapered (Fig 1b), which could lead to significant modifications to the spectral response [5]. In both theory and experiment, we see diameter independent Fabry-Perot type modulations in the visible wavelength range, and diameter-dependent guided light absorption at infrared wavelengths. Interestingly, the magnitude of the measured EQE is comparable to the simulated absorption, suggesting high internal quantum efficiencies (>80\%).

[1] L. Cao, P. Fan, E. S. Barnard, A. M. Brown and M. L. Brongersma, Nano Letters 10, 2649 (2010).

[2] K.Seo, M. Wober, P. Steinvurzel, E. Schonbrun, Y. Dan, T. Ellenbogen and K. B. Crozier, Nano letters 11, 1851 (2011).

[3] A. Solanki, P. Gentile, V. Calvo, G. Rosaz, B. Salem, V. Aimez, D. Drouin and N. Pauc, Nano Energy 1, 714 (2012).

[4] C. D. Tran, Anal. Lett. 38, 735 (2005).

[5] A. Solanki and K. B. Crozier, Appl. Phys. Lett. 105, 191115 (2014). 